\title{
A contoured nozzle design method for effective cold spray metal coating
}

\author{
Florentina-Luiza Zavalan*, Aldo Rona \\ School of Engineering, University of Leicester, Leicester, United Kingdom \\ *flz1@leicester.ac.uk
}

\begin{abstract}
In cold spraying, a uniform particle distribution of uniform velocity is desirable to achieve a good quality deposition pattern. Whereas many cold spray facilities use conical convergentdivergent nozzles for accelerating the particles, this paper evaluates a contoured axisymmetric nozzle design to enhance the process efficiency.

The performance of a conventional conical cold spray nozzle is compared with that of a new axisymmetric nozzle designed with a smooth throat and contoured for a parallel outflow. The new nozzle profile is obtained by the application of two aerospace design codes based on the Method of Characteristics. A two-way coupled Eulerian-Lagrangian Computational Fluid Dynamics formulation is used, where the continuous phase steady motion is predicted by solving the Reynolds-Averaged Navier-Stokes equations with the Shear Stress Transport k- $\omega$ model. A Discrete Phase Model computes the motion of the particles carried by the jet.

The numerical predictions show that the new nozzle shape delivers a more radially uniform deposition. A higher particle velocity is also obtained at the same operating conditions used by the industry standard nozzle, which is desirable for good particle bonding to the substrate.
\end{abstract}

\section{Keywords}

Cold spraying, metal coating, contoured nozzle design, Computational Fluid Dynamics, Discrete Phase Model.

\section{Introduction}

Cold spraying is increasingly attractive as a material coating technique, due to its potential applications to a variety of substrate materials [1]. Cold spraying consists in accelerating powder particles to a high velocity by a high speed flow. The particles impinge onto a substrate, where they plastically deform under the action of their kinetic energy and create a bond. The major advantage of this metal processing technique is the low temperature involved, which minimizes any potential phase change of the substrate and keeps the particles in their solid state. Alkhimov et al. [2] demonstrated that the key parameters for successful bonding of the particles with the substrate are the impact velocity and the temperature. A high velocity is necessary for a good deposition efficiency and packing density. At low temperatures, particle oxidation is avoided, which can make cold spray coatings more durable and with a better bond strength than equivalent plasma sprayed coatings, in some applications.

Given the key role of particle velocity and of particle kinetic energy on the quality of the cold spray coating, a good understanding of the factors that affect the particle velocity before impact is crucial for improving the current cold spray coating practices. Whereas many cold spray facilities use conical convergent-divergent nozzles for accelerating the particles, it is of interest to consider an alternative workflow to design contoured axisymmetric nozzles that enhance the radial uniformity of the velocity in the carrier phase. The significance of the nozzle design in the cold spray process is discussed by several authors in the literature, with some 
studies dedicated to the optimization of the cold spray nozzle configuration [3, 4]. Various nozzle shapes were investigated, including convergent-divergent nozzles [5-9], convergentdivergent-barrel nozzles [7], convergent-barrel nozzles [7, 10] and contoured axisymmetric (bell-shaped) nozzles [11, 12]. Furthermore, the effect of the nozzle cross-section on the particle velocity and distribution was investigated [13-16]. Other studies have shown that transonic particle drag limits the extent to which the particle velocity can be improved through nozzle redesign $[17,18]$. However, there are still some unanswered questions concerning the influence of the nozzle shape in cold spraying that warrant further investigation.

In this study, a three-dimensional Computational Fluid Dynamics (CFD) Reynolds-Averaged Navier-Stokes (RANS) model is developed to provide a preliminary assessment of the flow and particle behaviour in a lightly laden jet, in which well-dispersed titanium particles are accelerated by a compressible gas. ANSYS FLUENT ${ }^{\circledR}$ v19.5 is used as the CFD solver. The Shear Stress Transport (SST) $k-\omega$ turbulence closure model is used to account for the effect of turbulence on the time-averaged state of the continuous phase and the Discrete Phase Model (DPM) is used to compute the motion of the particles. The authors have redesigned the industrial nozzle investigated experimentally by Zahiri et al. [19]. A comparison of the performance of the original and redesigned nozzles is made for the same operating conditions and for the same particle size distribution.

\section{Numerical Methodology}

Nozzle design

In order to improve the acceleration of the particles through the conduit, a bell-shaped nozzle is proposed, to replace the conical convergent-divergent shape of the cold spray nozzles in current use by industry. This is achieved by adapting compressible flow design methods originally developed for transonic and supersonic wind tunnel nozzles. Bell-shaped nozzles produce a more uniform outflow, leading to a more uniform particle velocity distribution [11, 12]. They can prevent nozzle plugging or erosion by the spray. The goal is to improve the conventional nozzle geometry to produce higher quality flow at the same nozzle inflow conditions.

The design of the new nozzle is obtained by using the code developed by Alcenius and Schneider [20] in conjunction with the CONTUR code [21]. The code of Alcenius and Schneider, used for the convergent part, is based on the Hopkins and Hill [22] perturbation solution for the flow in the transonic region of an axisymmetric nozzle. CONTUR uses a combination of analytical solutions, the Method of Characteristics (MOC), and prescribed centerline distributions of axial velocity in order to define the divergent section profile of a convergent-divergent de Laval nozzle. This is a FORTRAN 77 code which runs through a series of 16 subroutines and uses seven user-defined input cards, which describe the desired flow conditions through the nozzle. A detailed description of the code is given in [21]. By combining the two codes, the entire axisymmetric nozzle contour shape is determined. A curvature-based design approach is used for coupling the geometrical profiles of the convergent and of the divergent sections, by which the curvature value from the CONTUR code is matched analytically and exactly at the throat, by scaling the curvature of the Alcenius and Schneider code profile output. The MOC used in this work departs from the minimumlength approach of aerospace nozzles to fit specific performance and economic constraints of cold spraying. Although the MOC nozzles have a more complex shape than conical nozzles, they do not present a significant manufacturing challenge with modern techniques [11, 12]. The new nozzle was designed by keeping the same inlet diameter, throat diameter and overall length as those of the nozzle it is based on, so it could seamlessly replace it on the cold spray 
commercial system. One of the critical design components of nozzle profile is the inflection angle. Supersonic MOC nozzles that produce uniform Mach number outflows are typically long with a small inflection angle, but there is no widely accepted value for this angle. For the design of the current nozzle, an inflection angle of $7^{\circ}$ was considered, based on Shope's recommendation to use inflection angles less than about $12^{\circ}$ to yield a uniform outflow [23]. A nozzle inlet convergent angle of $12^{\circ}$ was used, which is marginally steeper than the nozzle inlet convergent value of the baseline geometry. This small difference comes from the requirement of accommodating a radiused throat within the same nozzle length. The new nozzle has a throat curvature radius to throat radius ratio of 6 as in the sample nozzle design of Sivells [21] and as in the nozzle designed by Adams [24]. It is also close to the values of 5 and 6.6 used in [25]. This follows the recommendation by Sivells reported in [23] to use a large value for this parameter for flow quality, this value to be greater than 1 for accurate machining.

\section{Computational domain and boundary conditions}

The baseline numerical set-up consists of a conical convergent-divergent nozzle, namely, a nozzle manufactured for the CGT ${ }^{\mathrm{TM}} \mathrm{KINETIKS}^{\circledR} 4000$ cold spray system. The test case presented here uses the same properties for the carrier phase and for the dispersed phase as in Zahiri et al. [19]. This enables to build some confidence in the predictions of the gas flow state and of the solid particle kinetics by comparison with the work in [19]. The nozzle geometry details are presented in Table 1.

Table 1 - Nozzle geometry [19].

\begin{tabular}{l|c}
\hline Item & Size $[\mathrm{mm}]$ \\
\hline Inlet diameter & 18 \\
Throat diameter & 2.6 \\
Exit diameter & 8.5 \\
Pre-chamber length & 60 \\
Convergent section length & 51.2 \\
Divergent section length & 71.3
\end{tabular}

The nozzle discharge is modelled in the computational domain of Figure 1. A full threedimensional computation is performed, but for simplicity, Figure 1 shows only the half of the computational domain through the nozzle meridional plane.

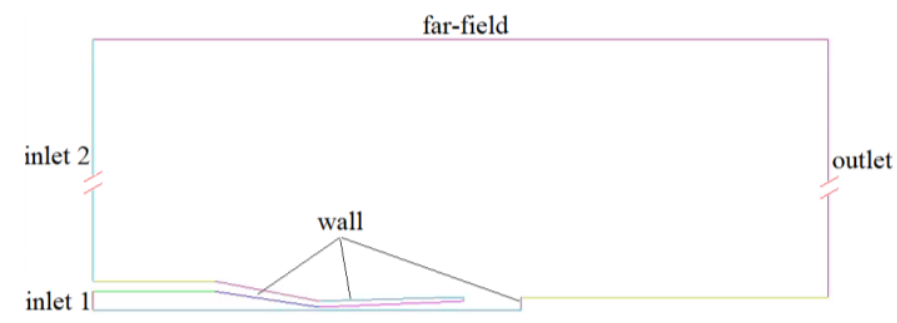

Figure 1. Sketch of the computational domain and boundaries.

The computational domain is obtained by revolving Figure 1 over $2 \pi$ about the nozzle axis. It has a diameter of $400 \mathrm{~mm}$ and a length of $361.5 \mathrm{~mm}$. Along the inlet boundaries, constant total pressure, $p_{0}$, and the total temperature, $T_{0}$, values are imposed. The nozzle is supplied with compressed nitrogen at $1.4 \mathrm{MPa}$ and $823.15 \mathrm{~K}$ and it discharges in air at $100 \mathrm{~Pa}$ gage and $288.15 \mathrm{~K}$. Nitrogen is modelled as a compressible ideal gas, at a constant specific heat 
ratio $C_{\mathrm{p}} / C_{\mathrm{v}}=1.4$. The specific heat capacity is varied with temperature to account for the significant adiabatic cooling that the gas undergoes upon expansion through the nozzle. The Andrews and Biblarz polynomial profile [26] is used to determine the specific heat capacity of nitrogen over the temperature range $250 \mathrm{~K} \leq T<1150 \mathrm{~K}$. The gas molecular viscosity is modelled by Sutherland's law. Titanium particles are injected at a constant rate of $0.2 \mathrm{~g} / \mathrm{s}$ with an initial particle velocity of $10 \mathrm{~m} / \mathrm{s}$ and an initial temperature of $298.15 \mathrm{~K}$. At the domain outlet, a constant pressure outflow condition is set, with zero gauge pressure. The far-field boundary was placed sufficiently far from the nozzle not to influence significantly its streamwise growth. At the nozzle walls and substrate, a no-slip adiabatic wall condition is applied together with the trap discrete phase boundary condition.

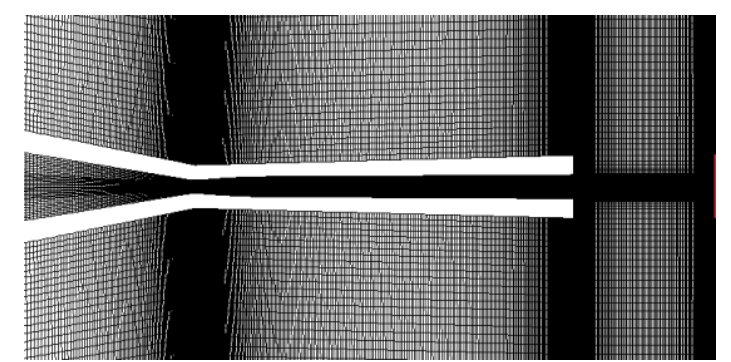

Figure 2. A zoomed-in view of the computational mesh around the cold spray nozzle.

The computational domain is discretized in finite volumes using a structured multi-block bodyfitted Cartesian mesh of about 4.5 million cells. This mesh is obtained by the commercial CFD mesh generator ICEM CFD ${ }^{\circledR}$ v19.5. Figure 2 shows the computational mesh detail around the nozzle, up to the substrate. Radial mesh clustering is used to resolve the nozzle wall boundary layer. Axial mesh clustering is applied at the throat, at nozzle exit, and close to the substrate, in order to capture the jet shear layer and the flow characteristics of the impinging jet. The spatial resolution reconciles the requirement of unit cells being no more than one order of magnitude larger than the largest particle size and of resolving the boundary layer over the nozzle wall. These requirements led to using a wall-normal cell size of $5.8 y+$ at the nozzle exit and a geometric mesh stretch factor of 1.05 .

\section{Numerical scheme}

The conservative laws of mass, momentum, energy, and species mass fraction are solved for the carrier phase using the pressure-based implicit flow solver in ANSYS FLUENT ${ }^{\circledR}$ v19.5. The steady-state flow solution method is used. The Semi-Implicit Method for Pressure Linked Equations (SIMPLE) scheme by Patankar [27] is used with a third-order MUSCL interpolation by van Leer [28] to determine the fluxes of the conservative laws at the finite-volume cell faces. Turbulence is accounted for by the SST $k-\omega$ model. The Mixture model is used to couple nitrogen and air. The motion of the titanium particles is computed using the DPM.

This model follows the two-way coupling approach: the fluid phase is treated as a continuum, by solving the RANS equations (Eulerian reference frame), and the dispersed phase is solved by tracking a number of particles through the calculated flow field of the continuous phase (Lagrangian reference frame).

Particles are assumed spherical, which is a reasonable approximation when particles are prepared using an appropriate milling process. The particle size distribution is approximated by a Rosin-Rammler distribution with the spread parameter of 3.7, as given in [19]. A sample of 7680 spherical particles is injected in the RANS converged flow solution from the prechamber inlet plane (inlet 1). The particle size in the sample ranges from $10 \mu \mathrm{m}$ to $70 \mu \mathrm{m}$. 
The particles are advanced by using the unsteady tracking method, which consists in calculating the trajectory of each particle by integrating the force balance on the particle. Three different drag laws are used for computing the drag coefficient of the particles, namely, the high-Mach-number drag law [29], Crowe's drag law [30] and Henderson's drag law [31]. The dispersion of the particles due to turbulent fluctuations in the flow is modelled using stochastic tracking, by the Discrete Random Walk model. This allows to predict the trajectories of particles using an estimated value of the instantaneous carrier phase (gas) velocity. A detailed description of these models can be found in the FLUENT User's Guide [32]. As the jet is lightly laden, particle-particle interactions are statistically rare and no collision model was used.

\section{Results and Discussion}

In this section, the results from the computational fluid dynamic simulations are organized as follows: first, the model validation for the discrete phase is presented and discussed; then, the profiles of the baseline nozzle and of the redesigned nozzle are presented and their effects on the gas velocity, particle velocity distribution, and on the particle radial spread are analysed.

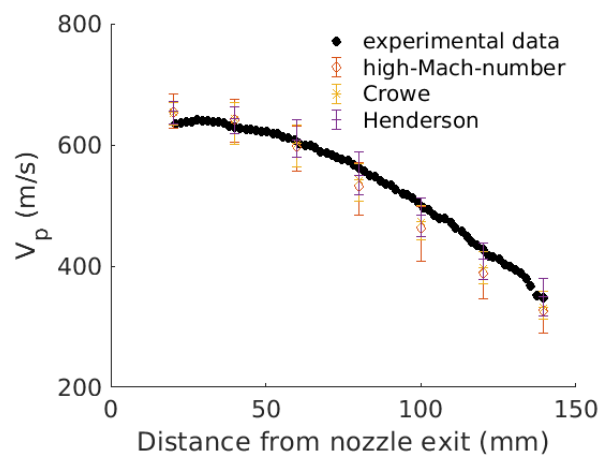

Figure 3. Comparison of titanium particle maximum speed as a function of distance from the nozzle exit [19].

Figure 3 shows the predicted titanium particle maximum speed as a function of distance from the nozzle exit. These predictions are compared against corresponding measurements from Zahiri et al. [19]. The maximum particle speed measured $20 \mathrm{~mm}$ from the nozzle exit is 640 $\mathrm{m} / \mathrm{s}$. The corresponding speed predicted by the three different drag laws is around $654 \mathrm{~m} / \mathrm{s}$, which is $2.19 \%$ higher. This comparison indicates that the current CFD model provides a close agreement to the experiment and it is therefore a good platform from which to evaluate the effect of nozzle design improvements. Measured and computed values of maximum particle speed are shown in Figure 3 to remain proximal to one another up to $60 \mathrm{~mm}$ from the nozzle exit. Further downstream, the agreement becomes coarser for the high-Mach-number and Crowe drag laws. However, current cold spray targets are typically placed up to $50 \mathrm{~mm}$ from the nozzle exit, therefore these targets would be within the region in which the drag laws retain a good predictive ability. On this basis, this model is taken onwards with the Henderson drag law, which provided slightly better agreement with the measurements over the entire experimental interval investigated, for the redesign of the nozzle.

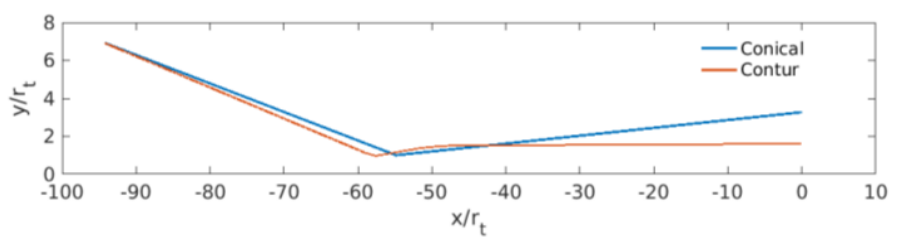

Figure 4. Sketch of the nozzle profiles. 
In Figure 4, the radial profiles of the internal wall of the conical convergent-divergent nozzle, by the blue line, and of the nozzle re-profiled using CONTUR, by the red line, are presented. The new nozzle has a convergent part $3.6 \mathrm{~mm}$ shorter than the baseline nozzle and an exit diameter of $4.7 \mathrm{~mm}$. The baseline convergent-divergent geometry is hereafter referred to as the Conical nozzle and the redesign output as the Contur nozzle.
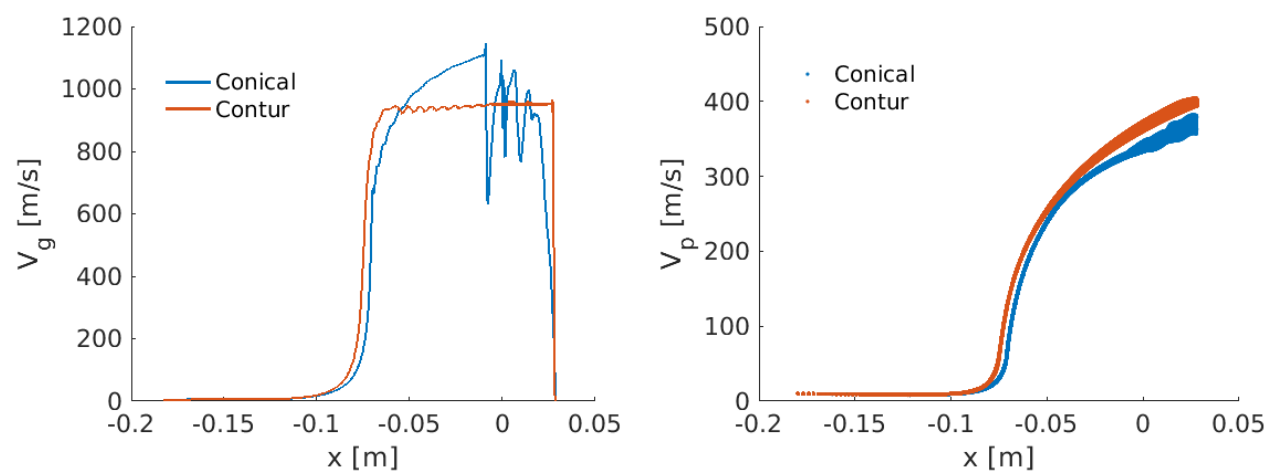

Figure 5. Velocity magnitude of (a) the carrier gas phase along the axis of the nozzle and of (b) titanium particles with a diameter of $70 \mu \mathrm{m}$.

Figure 5 (a) shows that the nozzle redesign significantly changed the velocity magnitude of the carrier gas phase. An earlier Mach number rise through the nozzle is obtained by locating the throat upstream, thereby exposing the titanium particles to high drift velocities sooner through the expanding gas. This is important, since the goal is to reduce the lag in the particle speed with respect to the gas speed at the nozzle exit, so that the particles can be accelerated above their critical velocity, which enables them to deposit on the substrate. The redesign has also essentially removed the shocks downstream of the Conical nozzle exit plane, up to the bow shock just in front of the substrate.

The speed change of a sample titanium particles with a diameter of $70 \mu \mathrm{m}$ is shown in Figure 5 (b). The titanium particles attain a higher velocity through the Contur nozzle than through the Conical baseline nozzle, while both nozzles are operating with the same gas supply. This indicates a two-fold advantage delivered by the redesign. On one hand, since the square of the peak particle speed is proportional to the particle kinetic energy, there is potential for a more energetic plastic deformation upon impact, which may improve the metal deposition characteristics. Additionally, the removal of lower speed particles may reduce waste of powder feed, since lower speed particles either rebound or poorly attach to the substrate.
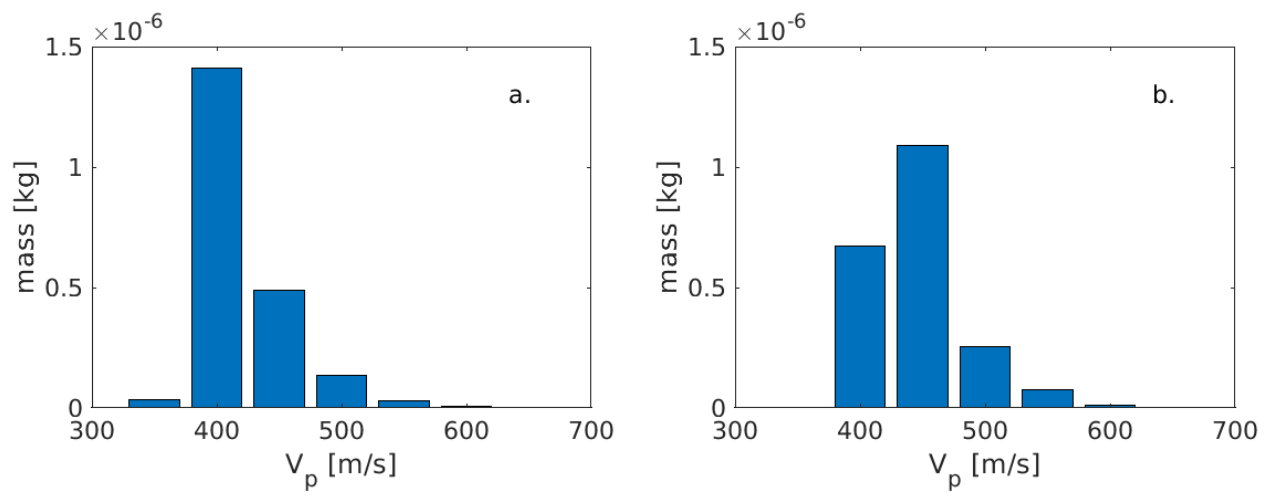

Figure 6. Mass weighted velocity distribution of titanium particles on impact with the substrate located at $28 \mathrm{~mm}$ from the nozzle exit (a) Conical nozzle and (b) Contur nozzle. 
This is also evidenced in Figure 6, where the mass weighted velocity distribution of titanium particles at $28 \mathrm{~mm}$ from the nozzle exit plane is shown. It can be observed that, by redesigning the nozzle, the particle speed distribution has changed, the titanium particles reaching a higher speed under the same conditions. For the particles in the distribution used, the Stokes number based on the nozzle exit diameter, particle relaxation time [33], and gas exit velocity ranges between 51 and 226, indicating an important particle lag to flow changes.
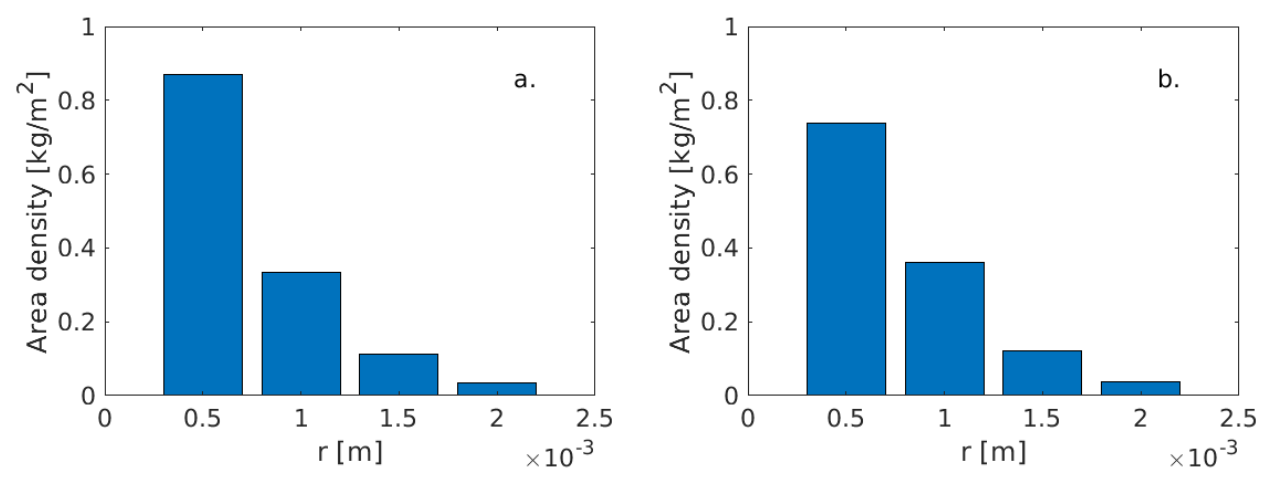

Figure 7. Area density of the titanium particles on impact with the substrate located at $28 \mathrm{~mm}$ from the nozzle exit (a) Conical nozzle and (b) Contur nozzle.

By redesigning the nozzle, the radial distribution of the particles is also affected by which particles are spread out more by the Contur nozzle. This can be observed in Figure 7, where the area density of titanium particles impacting the substrate is shown. The area density is determined by adding the mass of particles falling on concentric rings of area $2 \pi r \times 0.5 \mathrm{~mm}$, divided by each ring area. The baseline Conical nozzle is predicted to direct more particles radially close to the nozzle axis. This is likely to cluster the particles so that some of them will strike the substrate in the same place. Over time, this particle overlap will increase the angle of the deposited material, thereby creating deposits with a conical profile, rather than an even layer, the latter being more desirable in a metal spray deposition process. Therefore, the redesigned nozzle produces a more spread out and even particle impact pattern.

\section{Conclusions}

A numerical model of a nitrogen supersonic jet discharging in air, lightly laden by titanium particles, has shown the significance of the nozzle interior wall geometry on the particle motion. At a given nitrogen mass flow rate feed and nozzle pressure ratio, the axial change in the nozzle cross-section determines the acceleration of the expanding nitrogen. This controls the speed before impact of the titanium particles, in the modelled cold spray application. Performance benefits are achieved by improving the gas expansion by re-profiling the nozzle internal walls, compared to a commercial conical convergent-divergent cold spray nozzle of the same length, using the same gas feed inflow and nozzle pressure ratio.

The new nozzle wall profile is obtained by application of two aerospace design codes based on the MOC. The effectiveness of this redesign is tested numerically by CFD, using a two-way coupled Eulerian-Lagrangian formulation, in which the steady gas expansion is computed by a RANS SST $\mathrm{k}-\omega$ model and the particle motion is determined by DPM.

The new nozzle shape delivers a higher particle velocity at the same operating conditions used by the industry standard nozzle. A more radially uniform particle deposition profile is obtained, which is conducive to forming a more homogeneous metal coating. This nozzle 
design approach has excellent exploitation potential in cold spray systems as well as in threedimensional printing / additive layer manufacturing, to form better bonded coats and layers.

\section{Acknowledgments}

The PhD research of Florentina-Luiza Zavalan is funded and supported by EPSRC (EPSRC CDT Grant number EP/L016206/1) in Innovative Metal Processing. This research used the ALICE High Performance Computing Facility at the University of Leicester.

\section{References}

[1] Moridi, A., Hassani-Gangaraj, S.M., Guagliano, M., Dao, M., 2014, Surf. Eng., 36, pp. 369395.

[2] Alkhimov, A.P., Kosareve, V.F., Papyrin, A.N., 1990, Dokl. Akad. Nauk SSSR, 315, pp. $1062-1065$

[3] Dykhuizen, R.C., Smith, M.F., 1998, J. Therm. Spray Technol., 7(2), pp. 205-212.

[4] Alkhimov, A.P., Kosareve, V.F., Papyrin, A.N., 2001, J. Therm. Spray Technol., 10(2), pp. 375-381.

[5] Sakaki, K., Shimizu, Y., 2001, J. Therm. Spray Technol., 10(3), pp. 487-496.

[6] Stoltenhoff, T., Kreye, H., Richter, H.J., 2002, J. Therm. Spray Technol., 11, pp. 542-550.

[7] Sakaki, K., Huruhashi, N., Tamaki, K., Shimizu, Y., March 2002, International Thermal Spray Conference.

[8] Li, W.Y., Li, C.J., 2005, J. Therm. Spray Technol., 14(3), pp. 391-396.

[9] Heinrich, P., Kreye, H., Stoltenhoff T., 2005, U.S. Patent 2005/0001075 A1.

[10] Li, W.Y., Liao, H., Wang, H.T., Li, C.J., Zhang G., Coddet, C., 2006, Appl. Surf. Sci., 253(2), pp. 708-713.

[11] Gärtner, F., Stoltenhoff, T., Schmidt, T., Kreye, H., 2006, J. Therm. Spray Technol., 15(2), pp. 223-232.

[12] Schmidt, T., Assadi, H., Gärtner, F., Richter, H., Stoltenhoff, T., Kreye, H., Klassen, T., 2009, J. Therm. Spray Technol., 18(5-6), pp. 794-808.

[13] Tabbara, H., Gu, S., McCartney, D.G., Price, T.S., Shipway, P.H., 2011, J. Therm. Spray Technol., 20(3), pp. 608-620.

[14] Yin, S., Wang, X.F., Li, W.Y., 2011, Surf. Coatings Technol., 205, pp. 2970-2977.

[15] Suo, X.K., Liu, T.K., Li, W.Y., Suo, Q.L. Planche, M.P., Liao, H.L., 2013, Surf. Coatings Technol., 220, pp. 107-111.

[16] Varadaraajan, V., Mohanty, P., 2017, Surf. Coatings Technol., 316, pp. 246-254.

[17] Jodoin, B., 2002, J. Therm. Spray Technol., 11(4), pp. 496-507.

[18] Grujicic, M., DeRosset, W.S., Helfritch, D., 2003, Proc. Inst. Mech. Eng., 217, pp. 1-11.

[19] Zahiri, S.H., Yang, W., Jahedi, M., 2009, J. Therm. Spray Technol., 18, pp 110-117.

[20] Alcenius, T.J., Schneider, S.P., 1994, NASA-CR-194857.

[21] Sivells, J.C., 1978, ARO Inc., ADEC-TR-78-63.

[22] Hopkins, D.F., Hill, D.E., 1966, AIAA Journal, 4(8), pp. 1337-1343.

[23] Shope, F.L., June 2006, AIAA-2006-3665, 24th Applied Aerodynamics Conference.

[24] Adams, S.E., 2016, "The Design and Computational Validation of a Mach 3 Wind Tunnel Nozzle Contour" Master thesis.

[25] Alcenius, T.J., Schneider, S.P., Beckwith, I.E., White, J.A., Korte, J.J., 1996, J. Aircr., 33(6), pp. 1131-1138.

[26] Andrews, J.R., Biblarz, O., 1981, Naval Postgraduate School, NPS67-81-001.

[27] Patankar, S.V., 1980, "Numerical Heat Transfer and Fluid Flow".

[28] Van Leer, B., 1974, J. Comput. Phys., 14, pp. 361-370.

[29] Clift, R., Grace, J.R., Weber, M.E., 1978, "Bubbles, Drops, and Particles".

[30] Crowe, C.T., 1967, AIAA Journal, 5, pp. 1021-1022.

[31] Henderson, C.B., 1976, AIAA Journal, 14, pp. 707-708.

[32] Ansys Inc., 2012, "ANSYS FLUENT Theory Guide".

[33] Gosman, A.D., loannides, E., 1983, J. Energy, 7(6), pp. 482-490. 